\title{
Anomalies in curved spacetime at finite temperature
}

\author{
H. Boschi-Filho \\ Instituto de Física, Universidade Federal do Rio de Janeiro, 21945 Rio de Janeiro, Caixa Postal 68528, Rio de Janeiro, Brazil \\ and Departamento de Física e Química, Universidade Estadual Paulista, Campus de Guaratinguetá, \\ 12500 Guaratinguetá, Caixa Postal 205 São Paulo, Brazil \\ C. P. Natividade \\ Departamento de Matemática, Universidade Estadual Paulista, Campus de Guaratinguetá, 12500 Guaratinguetá, \\ Caixa Postal 205, São Paulo, Brazil
}

(Received 22 May 1992)

\begin{abstract}
We discuss the problem of the breakdown of conformal and gauge symmetries at finite temperature in curved-spacetime background, when the changes in the background are gradual, in order to have a welldefined quantum field theory at finite temperature. We obtain the expressions for Seeley's coefficients and the heat-kernel expansion in this regime. As applications, we consider the self-interacting $\lambda \phi^{4}$ and chiral Schwinger models in curved backgrounds at finite temperature.
\end{abstract}

PACS number(s): 03.70. $+\mathrm{k}, 04.60 .+\mathrm{n}, 11.15 . \mathrm{Tk}, 11.30 . \mathrm{Rd}$

\section{INTRODUCTION}

The problem of determining the anomalies in quantum field theory at finite temperature, both in flat and curved spacetimes, has been studied by many authors [1-11]. Pioneering work, on flat spacetime, was developed by Dolan and Jackiw [1] showing that the polarization tensor and so the anomalous divergence of the axial-vector current, for two-dimensional QED, is the same at any finite temperature. This result was generalized to all orders of the perturbation theory and to non-Abelian gauge fields by Itoyama and Mueller [2]. Then, many other works using different techniques and models to that anomalous behavior at finite temperature in a flat spacetime have appeared in the literature $[3,4]$. In particular, the anomaly for a derivative coupling model by using the Schwinger-DeWitt expansion $[12,13]$ at finite temperature has been calculated recently [4].

In addition, quantum field theory in a curved background has been extensively studied and the role of conformal invariance was regained from the classical context to these theories. It was soon realized that conformal invariance, especially the invariance of the trace of the energy-momentum stress tensor, would be broken in quantized theories, even at the one-loop level [13]. So the question of restoration of this symmetry at finite temperatures was investigated. This was realized for conformal fields in static (and ultrastatic) spacetimes, since in these cases the conditions for thermal equilibrium can be assumed and some applications to particular universes were possible [5-7]. The applications of these results to conformally static spacetimes, such as the Robertson-Walker universes, were also done [8-10] and the discussion of quasiequilibrium conditions for nearly conformal fields were put forward first by Drummond [9] in a version of the imaginary time formalism [14] and after by Semenoff and Weiss [10] using real time techniques. The extension of these works to a general curved background were dis- cussed by $\mathrm{Hu}$ and co-workers [11] imposing gradual changes in the background metric and fields. They used momentum-space techniques and a quasilocal expansion to derive the imaginary time thermal Green's functions and the one-loop finite-temperature effective Lagrangian for $\lambda \phi^{4}$ fields in curved spacetimes.

In this paper we are going to discuss anomalous behavior in quantum field theory at finite temperature in curved spacetimes as established by $\mathrm{Hu}$ and co-workers [11], i.e., assuming that only gradual changes occur in the backgrounds, in order to guarantee that quasiequilibrium conditions hold. As we are especially interested in the anomalous behavior of field theories at finite temperatures and regularization schemes play a central role in the calculation of anomalies, we must use a regularization technique adequate for finite temperature. This is done in Sec. II, for the heat-kernel expansion which is suitable for regulating theories both in flat and curved spacetimes. Moreover, with the heat-kernel expansion it is easy to obtain the generalized $\zeta$ function which has also been largely used as a regulator $[13,15]$. In a previous work [4], the asymptotic expansion of the heat kernel at finite temperature was obtained in flat spacetime. In the present work, we will generalize that previous results to curved spacetimes.

With this expansion we discuss, in Sec. III, the selfinteracting $\lambda \phi^{4}$ scalar field at finite temperature in a curved background. This is done in a semiclassical approximation, which is equivalent to the theory at the one-loop level [16-18]. The results obtained in this work reduce to the previous ones when we take the zerotemperature limit, as was expected. In fact, we show that the trace anomaly for a curved background is temperature independent, in accordance with the results in static or quasistatic [5-7] and also conformally flat spacetimes [8].

This behavior is very close to that of the axial anomaly, which has been shown to be temperature independent for 
some models in flat spacetime [ $1-3]$. This fact motivated us to discuss the chiral Schwinger model $[19,20]$, whose flat spacetime features are well known. Since previous attempts to calculate the anomalous divergence of the axial-vector current and the dynamically generated mass, in curved spacetime [21], did not give results consistent with the flat spacetime ones, we discuss first this model in curved spacetime at zero temperature obtaining the correct anomalous current and mass. Then, we introduce the imaginary time formalism $[9,11,14]$ and discuss the anomalous behavior at finite temperatures in the case of a curved background. It is shown that the anomaly remains the same at all finite temperatures. This will be discussed in detail in Sec. IV and some topological aspects related to the axial anomaly will be also commented on Sec. V.

\section{THE HEAT-KERNEL EXPANSION AT FINITE TEMPERATURE}

In order to determine the chiral and trace anomalies via Fujikawa's method $[22,23]$ in a thermal average, at finite temperature $T=\beta^{-1}$, we will evaluate the asymptotic expansion of the heat kernel at this temperature $\beta^{-1}$. The two problems that arise when we try to make the extension of the flat spacetime results [4] are the momentum representation is not free from ambiguities in curved spacetime and thermal equilibrium is only strictly maintained for massless conformally coupled fields in conformally static backgrounds.

The first question has been addressed by Bunch and Parker [24] who showed that a local-momentum representation for propagators is possible with the use of Riemann normal coordinates, since their behavior at each spacetime point can be locally described by flat spacetime, according to the equivalence principle. An equivalent approach, which we will use in this paper, is given by the local frame vierbein fields $e^{a}{ }_{\mu}$. In fact, as anomalies involve only high momenta and short wavelengths, this works precisely to validate the asymptotic expansion of the heat kernel in curved spacetime.

The second question has not been solved for arbitrary spacetimes but only for nearly conformal static background metrics and nearly conformal background fields by $\mathrm{Hu}$ and co-workers [11]. In their approach they succeed to derive thermal Green's functions via momentum space techniques and a quasilocal expansion in backgrounds which present gradual changes in the background metric and background fields. These approximations were useful to describe quasiequilibrium conditions and permitted the discussion of universes with small rates of expansion compared with the adiabatic parameter, defined by $\mathrm{Hu}[11]$, which measures the departure from the adiabacity for any mode with a given frequency and also for small anisotropic background metrics.

In addition, it was pointed out by $\mathrm{Hu}$ and co-workers that the equivalence of small proper-time expansion (heat-kernel technique) and adiabatic methods results in a well-defined finite-temperature quantum field theory. With this established we can construct the heat-kernel expansion at finite temperature unambiguously. With this expansion at hand we can obtain the anomaly in $N$ (even-) dimensional spacetime, as usual, from the corresponding Seeley coefficient $a_{N / 2}[12,13,25]$.

Let us start with the definition of the heat kernel in $N$ dimensional spacetime at zero temperature [12],

$$
H^{(N)}\left(x, x^{\prime} ; t\right)=\left\langle x^{\prime}\left|e^{-t D^{2}}\right| x\right\rangle,
$$

which satisfies the heat equation

$$
\left(\frac{\partial}{\partial t}+D^{2}\right) H^{(N)}\left(x, x^{\prime} ; t\right)=0,
$$

with the boundary condition

$$
H^{(N)}\left(x, x^{\prime} ; 0\right)=\left\langle x^{\prime} \mid x\right\rangle,
$$

where

$$
D^{2}=D^{\mu} D_{\mu}+X
$$

$D_{\mu}$ is the Dirac operator and $X$ is a function which depends on the specific choice of $D_{\mu}$, but contains no derivatives. The orthonormality condition can be written in curved spacetime, at zero temperature, as

$$
\left\langle x^{\prime} \mid x\right\rangle=V^{1 / 2}\left(x, x^{\prime}\right) I\left(x, x^{\prime}\right) \delta^{(N)}\left(x, x^{\prime}\right),
$$

where $V\left(x, x^{\prime}\right)$ is the Van Vleck-Morette determinant $[12,13]$

$$
V\left(x, x^{\prime}\right)=\operatorname{det}\left(\sigma\left(x, x^{\prime}\right)_{; \mu v^{\prime}}\right),
$$

which satisfies

$$
V^{1 / 2} \sigma_{; \mu}^{\mu}+2 \sigma^{; \mu} V_{; \mu}^{1 / 2}=N V^{1 / 2}
$$

Half of the square of the geodesic distance between $x$ and $x^{\prime}, \sigma\left(x, x^{\prime}\right)$, and the geodetic parallel transport $I\left(x, x^{\prime}\right)$ satisfies

$$
\sigma^{; \mu} I_{; \mu}=\sigma^{; v^{\prime}} I_{; v^{\prime}}=0
$$

with the boundary condition $I(x, x)=1$. Now, to define the heat kernel at finite temperature we extend the relation $(2.4)$ to $[9,26]$

$$
\left\langle x^{\prime} \mid x\right\rangle_{\beta}=V^{1 / 2}\left(x, x^{\prime}\right) I\left(x, x^{\prime}\right) \delta_{\beta}^{(N)}\left(x, x^{\prime}\right),
$$

since the quantities $V\left(x, x^{\prime}\right)$ and $I\left(x, x^{\prime}\right)$ are of a geometrical nature and may be not changed by the thermal average. On the other hand, the generalized delta function $\delta_{\beta}^{(N)}\left(x, x^{\prime}\right)$ must exhibit the same periodicity conditions as the eigenfunctions of the operator $D^{2}$, so [9]

$$
\begin{array}{r}
\delta_{\beta}^{(N)}\left(x, x^{\prime}\right)=\sum_{n=-\infty}^{+\infty} \int \frac{d^{(N)} k}{(2 \pi)^{N}} e^{i k_{\sigma\left(x, x^{\prime}\right)} ; \mu}\left(\frac{2 \pi}{\beta}\right) \\
\times \delta\left[k_{0}-\frac{2 \pi}{\beta}\left(n+\frac{1}{2}\right)\right],
\end{array}
$$

in close analogy with the $\delta$-function expansion in flat spacetime at finite temperature [4]. This choice of the $\delta$ function corresponds to antiperiodic Fermi fields relative to time coordinate. The corresponding $\delta$ function for boson fields can be obtained simply substituting $n+\frac{1}{2}$ by $n$, as will be discussed in Sec. III. Using this definition we can rewrite the heat kernel (2.1), at finite temperature, as 


$$
\begin{aligned}
H_{\beta}^{(N)}\left(x, x^{\prime} ; t\right) & =\exp \left(-t D^{2}\right)\left\langle x^{\prime} \mid x\right\rangle_{\beta} \\
& =\sum_{n=-\infty}^{+\infty} \int \frac{d^{(N)} k}{(2 \pi)^{N}} \exp \left(-t D^{2}\right) \exp \left[i k^{\mu} \sigma\left(x, x^{\prime}\right)_{; \mu}\right]\left[\frac{2 \pi}{\beta}\right] \delta\left[k_{0}-\frac{2 \pi}{\beta}\left(n+\frac{1}{2}\right)\right] V^{1 / 2}\left(x, x^{\prime}\right) I\left(x, x^{\prime}\right) .
\end{aligned}
$$

Now, following Yajima [27], who has evaluated the heat kernel for spin- $\frac{1}{2}$ fields at zero temperature in curved spacetime using local-momentum representation, we take the diagonal part $\left(x=x^{\prime}\right)$ of Eq. (2.9) and rewrite the exponentials involving $D^{2}$ and $\sigma\left(x, x^{\prime}\right)$ to find

$$
\begin{aligned}
& H_{\beta}^{(N)}(x, x ; t)=\sum_{n=-\infty}^{+\infty} \int \frac{d^{(N)} k}{(2 \pi \sqrt{t})^{N}}\left\lceil\left[ e^{k^{\mu} k_{\mu}} \mid 1-t\left[D_{\mu} D^{\mu}+X+k^{\rho} k^{\tau}\left(\sigma_{; \rho \tau \mu}^{\mu}+\sigma_{; \rho \mu}{ }^{\mu}+2 D_{\rho} D_{\tau}\right)\right]\right.\right. \\
& +t^{2}\left\{\frac{1}{2} D_{\mu} D^{\mu} D_{i} D^{v}+\frac{1}{2} X_{; \mu}{ }^{\mu}+\frac{1}{2} X^{2}+X D_{\mu} D^{\mu}\right. \\
& +k^{\rho} k^{\tau}\left[\frac{2}{3}\left(D_{\rho} D_{\tau} D_{\mu} D^{\mu}+D_{\rho} D_{\mu} D^{\mu} D_{\tau}+D_{\mu} D^{\mu} D_{\rho} D_{\tau}\right)\right. \\
& +\left(\sigma_{; \rho \mu}{ }^{\mu}{ }_{\tau}+\sigma_{; \rho \tau \mu}{ }^{\mu}\right) D_{v} D^{v}+\frac{4}{3}\left(2 \sigma_{; \rho \mu \nu \tau}+\sigma_{; \rho \tau \nu \mu}\right) D^{v} D^{\mu} \\
& +\frac{2}{3}\left(2 \sigma_{; \rho \mu \nu}{ }^{v}+\sigma_{; \rho v \mu}{ }^{v}\right) D_{\tau} D^{\mu}+\frac{2}{3}\left(\sigma_{; \rho \mu \nu}{ }^{v}+2 \sigma_{; \rho v \mu}{ }^{v}\right) D^{\mu} D_{\tau} \\
& +\frac{1}{3}\left(\sigma_{; \rho \tau \mu}^{\mu \nu}{ }^{v}+\sigma_{; \rho \mu}^{\mu v}{ }_{v \tau}+\sigma_{; \rho \mu}^{\mu}{ }^{\mu}{ }^{v}\right)+\frac{2}{3} \sigma_{; \rho \mu \nu \xi} \sigma_{; \tau}{ }^{\mu \nu \xi} \\
& +\frac{1}{3}\left(\sigma_{; \rho \mu \nu}{ }^{v} \sigma_{; \tau}{ }^{\mu \xi} \xi_{\xi}+\sigma_{; \rho \mu \nu}{ }^{v} \sigma_{; \tau \xi}{ }^{\xi \mu}+\sigma_{; \rho \mu}{ }^{\mu \xi} \sigma_{; \tau v}{ }^{v}\right) \\
& \left.+\frac{2}{3} X_{; \rho \tau}+2 X D_{\rho} D_{\tau}+\left(\sigma_{; \rho \mu}^{\mu}{ }_{\tau}+\sigma_{; \rho \tau \mu}{ }^{\mu}\right) X\right] \\
& +k^{\rho} k^{\tau} k^{\gamma} k^{\delta}\left[\frac{2}{3} D_{\rho} D_{\tau} D_{\gamma} D_{\delta}+2\left(\sigma_{; \rho \mu}^{\mu}{ }_{\tau}+\sigma_{; \rho \tau \mu}{ }^{\mu}\right) D_{\gamma} D_{\delta}\right. \\
& +\frac{1}{3}\left(\sigma_{; \rho \mu}^{\mu}{ }_{\tau \gamma \delta}+\sigma_{; \rho \tau \mu}^{\mu}{ }_{\gamma \delta}+\sigma_{; \rho \tau \gamma \mu}^{\mu}{ }_{\delta}+\sigma_{; \rho \tau \gamma \delta \mu}{ }^{\mu}\right) \\
& +\frac{2}{3}\left(\sigma_{; \rho \tau \mu \nu} \sigma_{; \gamma \delta}^{\mu v}+2 \sigma_{; \rho \mu \tau v} \sigma_{; \gamma \delta}{ }^{\mu v}+3 \sigma_{; \rho \mu \tau \nu} \sigma_{; \gamma}{ }^{\mu \nu}{ }^{\nu}\right) \\
& \left.\left.\left.+\frac{1}{2}\left(\sigma_{; \rho \tau \mu}^{\mu}+\sigma_{; \rho \mu}^{\mu}{ }_{\tau}\right)\left(\sigma_{; \gamma \delta v}{ }^{v}+\sigma_{; \gamma \nu \delta}{ }^{v}\right)\right]\right\}+O\left(t^{3}\right)\right) \\
& \left.\left.\times\left(\frac{2 \pi \sqrt{t}}{\beta}\right] \delta\left[k_{0}-\frac{2 \pi \sqrt{t}}{\beta}\left(n+\frac{1}{2}\right)\right] V^{1 / 2}\left(x, x^{\prime}\right) I\left(x, x^{\prime}\right)\right]\right],
\end{aligned}
$$

Observe that we assume that the limit $x^{\prime} \rightarrow x$ [represented by the double square brackets $[[\cdots]]$, on the right-hand side of Eq. (2.10)] is taken after the action of the differential operators $D_{\mu}$ on the quantities $V\left(x, x^{\prime}\right)$ and $I\left(x, x^{\prime}\right)$. In order to obtain the asymptotic expansion of the heat kernel at finite temperature, i.e., the generalized Seeley coefficients, we must sum Eq. (2.10) over $n$ and integrate it over $k$. To do so we will use the generalized $\Theta$-function transformation $[4,28]$ :

$$
\left.\left.\left.\sum_{n=-\infty}^{+\infty}\left[4 \pi^{2} \gamma\left(n+\frac{1}{2}\right)^{2}\right]^{p} e^{-4 \pi^{2} \gamma(n+1 / 2)^{2}}=\frac{1}{\sqrt{\pi \gamma}} \frac{(2 \rho-1) ! !}{2^{p+1}}\right] 1+2 \sum_{n=1}^{\infty}(-1)^{n} e^{-n^{2} / 4 \gamma} \sum_{l=0}^{p}\left[-\frac{n^{2}}{4 \gamma}\right]^{l} C\right]^{p}\right],
$$

where

$$
C_{l}^{p}=\frac{2^{l} p !}{l !(p-l) !(2 l-1) ! !}
$$

and the Gaussian integrals

$$
\begin{aligned}
& \int d^{(N)} k e^{-k^{2}}=(\pi)^{N / 2}, \\
& \int d^{(N)} k e^{-k^{2}} k_{\mu} k_{v}=-\frac{1}{2}(\pi)^{N / 2} g_{\mu v}, \\
& \int d^{(N)} k e^{-k^{2}} k_{\mu} k_{v} k_{\rho} k_{\tau}=\frac{1}{4}(\pi)^{N / 2}\left(g_{\mu \nu} g_{\rho \tau}+g_{\mu \rho} g_{v \tau}+g_{\mu \tau} g_{v \rho}\right) .
\end{aligned}
$$

Combining Eqs. (2.11) and (2.12) we have

$$
\sum_{n=-\infty}^{+\infty} \int d^{(N)} k e^{-k^{2}} \delta\left(k_{0}-2 \pi \sqrt{\gamma}\left(n+\frac{1}{2}\right)\right)=\frac{1}{2} \frac{(\pi)^{(N-1) / 2}}{\sqrt{\gamma}} S_{0}^{F}(1 / \gamma)
$$




$$
\begin{gathered}
\sum_{n=-\infty}^{+\infty} \int d^{(N)} k e^{-k^{2}} k_{\rho} k_{\tau} \delta\left(k_{0}-2 \pi \sqrt{\gamma}\left(n+\frac{1}{2}\right)\right)=-\frac{1}{4} \frac{(\pi)^{(N-1) / 2}}{\sqrt{\gamma}}\left[g_{\rho \tau} S_{0}^{F}(1 / \gamma)+g_{\rho 0} g_{\tau 0} S_{1}^{F}(1 / \gamma)\right] \\
\sum_{n=-\infty}^{+\infty} \int d^{(N)} k e^{-k^{2}} k_{\rho} k_{\tau} k_{\eta} k_{\xi} \delta\left(k_{0}-2 \pi \sqrt{\gamma}\left(n+\frac{1}{2}\right)\right) \\
=\frac{1}{8} \frac{(\pi)^{(N-1) / 2}}{\sqrt{\gamma}}\left[\left(g_{\rho \tau} g_{\eta \xi}+g_{\rho \eta} g_{\tau \xi}+g_{\rho \xi} g_{\tau \eta}\right) S_{0}^{F}(1 / \gamma)+\left(g_{\rho \tau} g_{\eta 0} g_{\xi 0}+g_{\rho \eta} g_{\tau 0} g_{\xi 0}+g_{\rho \xi} g_{\tau 0} g_{\eta 0}+g_{\tau \eta} g_{\rho 0} g_{\xi 0}\right.\right. \\
\left.\left.\quad+g_{\tau \xi} g_{\rho 0} g_{\eta 0}+g_{\eta \xi} g_{\rho 0} g_{\tau 0}\right) S_{1}^{F}(1 / \gamma)+g_{\rho 0} g_{\tau 0} g_{\eta 0} g_{\xi 0} S_{2}^{F}(1 / \gamma)\right]
\end{gathered}
$$

where

$$
\begin{aligned}
& S_{0}^{F}(1 / \gamma)=1+2 \sum_{n=1}^{+\infty}(-1)^{n} \exp \left(-n^{2} / 4 \gamma\right), \\
& S_{1}^{F}(1 / \gamma)=\frac{1}{2} \sum_{n=1}^{+\infty}(-1)^{n} \frac{n^{2}}{\gamma} \exp \left(-n^{2} / 4 \gamma\right), \\
& S_{2}^{F}(1 / \gamma)=\frac{1}{2} \sum_{n=1}^{+\infty}(-1)^{n}\left[\frac{n^{2}}{\gamma}\right]^{2} \exp \left(-n^{2} / 4 \gamma\right),
\end{aligned}
$$

stand for the fermion fields, since Eq. (2.11) must be modified to treat boson fields. Using Eq. (2.13a) in (2.10) we can find the zeroth-order term in $t$ of the heat-kernel expansion:

$$
\sum_{n=-\infty}^{+\infty} \int \frac{d^{(N)} k}{(2 \pi \sqrt{t})^{N}} \exp \left[k^{\mu} k_{\mu}\right]\left(\frac{2 \pi \sqrt{t}}{\beta}\right) \delta\left(k_{0}-\frac{2 \pi \sqrt{t}}{\beta}\left(n+\frac{1}{2}\right)\right] V^{1 / 2}\left(x, x^{\prime}\right) I\left(x, x^{\prime}\right)=\frac{e}{(4 \pi t)^{N / 2}} S_{0}^{F}\left(\beta^{2} / t\right),
$$

where $e=\lim _{x^{\prime} \rightarrow x} V^{1 / 2}\left(x, x^{\prime}\right) \equiv\left[V^{1 / 2}\right]$. Taking the coefficients of order $t$ in Eq. (2.10) and applying Eqs. (2.13a) and (2.13b) we find

$$
\begin{gathered}
-\sum_{n=-\infty}^{+\infty} \int \frac{d^{(N)} k}{(2 \pi \sqrt{t})^{N}} \exp \left(k^{\mu} k_{\mu}\right)\left[D_{\mu} D^{\mu}+X+k^{\rho} k^{\tau}\left(\sigma_{; \rho \tau \mu}{ }^{\mu}+\sigma_{; \rho \mu}{ }^{\mu}+2 D_{\rho} D_{\tau}\right)\right] \\
\times\left(\frac{2 \pi \sqrt{t}}{\beta}\right) \delta\left(k_{0}-\frac{2 \pi \sqrt{t}}{\beta}\left(n+\frac{1}{2}\right)\right] V^{1 / 2}\left(x, x^{\prime}\right) I\left(x, x^{\prime}\right) \\
=-\frac{1}{(4 \pi t)^{N / 2}}\left\{\left[D_{\mu} D^{\mu}+X-\frac{1}{2}\left(\sigma_{; \mu}{ }^{\mu}{ }_{v}{ }^{\nu}+\sigma_{; \mu \nu}{ }^{\nu \mu}+2 D_{\mu} D_{\mu}\right)\right] S_{0}^{F}\left(\beta^{2} / t\right)\right. \\
\left.-\frac{1}{2}\left[\sigma_{; 00 \mu}{ }^{\mu}+\sigma_{; 0 \mu}{ }^{\mu 0}+2 D_{0}{ }^{2}\right] S_{1}\left(t / \beta^{2}\right)\right\} V^{1 / 2}\left(x, x^{\prime}\right) I\left(x, x^{\prime}\right) \\
=\frac{e \quad}{(4 \pi t)^{N / 2}}\left(\frac{1}{6} R-X\right) S_{0}^{F}\left(\beta^{2} / t\right),
\end{gathered}
$$

where we have used the coincidence limits

$$
\left[V_{; \mu \nu}^{1 / 2}\right]=-\frac{1}{6} e R_{\mu \nu}, \quad\left[\sigma_{; \rho \mu}^{\tau}{ }^{\mu}\right]=\frac{2}{3} R_{\rho}^{\tau}, \quad\left[\sigma_{; \rho \mu}^{\mu \tau}\right]=-\frac{1}{3} R_{\rho}^{\tau}
$$

Now, taking the coefficients of $t^{2}$ in Eq. (2.10), using (2.13a)-(2.13c) and various coincidence limits involving $\sigma\left(x, x^{\prime}\right), V^{1 / 2}\left(x, x^{\prime}\right)$ and $I\left(x, x^{\prime}\right)[12,29]$, we find

$$
\begin{gathered}
\frac{1}{(4 \pi t)^{N / 2}}\left\{\frac{1}{12}\left[D_{\mu}, D_{v}\right]\left[D^{\mu}, D^{v}\right]+\frac{1}{6} X_{; \mu}{ }^{\mu}+\frac{1}{2} X^{2}-\frac{1}{6} R X-\frac{1}{30} R_{; \mu}{ }^{\mu}+\frac{1}{72} R^{2}\right. \\
\left.+\frac{1}{180}\left(R_{\mu \nu \rho \tau} R^{\mu \nu \rho \tau}-R_{\mu \nu} R^{\mu v}\right)\right\} V^{1 / 2}\left(x, x^{\prime}\right) I\left(x, x^{\prime}\right) S_{0}^{F}\left(\beta^{2} / t\right) \\
=\frac{e}{(4 \pi t)^{N / 2}}\left[\frac{1}{12} \Lambda_{\mu v} \Lambda^{\mu v}+\frac{1}{180}\left(R_{\mu \nu \rho \tau} R^{\mu \nu \rho \tau}-R_{\mu v} R^{\mu v}\right)-\frac{1}{6}\left(\frac{1}{5} R-X\right)_{; \mu}{ }^{\mu}+\frac{1}{2}\left(\frac{1}{6} R-X\right)^{2}\right] S_{0}^{F}\left(\beta^{2} / t\right),
\end{gathered}
$$


where

$$
\Lambda_{\mu v}=-\frac{1}{4} i R_{\mu \nu}^{a b} \sigma_{a b}+i F_{\mu v} .
$$

Finally collecting the results (2.15), (2.16), and (2.18) we can write the first few terms of the asymptotic expansion of the heat kernel for spin- $\frac{1}{2}$ fields at finite temperature as

$$
\begin{aligned}
H_{\beta}^{(N)}(x, x ; t)=\frac{e}{(4 \pi t)^{N / 2}}[ & a_{0}(x)+a_{1}(x) t+a_{2}(x) t^{2} \\
& \left.+O\left(t^{3}\right)\right] S_{0}^{F}\left(\beta^{2} / t\right)
\end{aligned}
$$

where

$$
\begin{aligned}
a_{0}(x)= & 1 \\
a_{1}(x)= & \frac{1}{6} R-X \\
a_{2}(x)= & \frac{1}{12} \Lambda_{\mu v} \Lambda^{\mu v}+\frac{1}{180}\left(R_{\mu v \rho \tau} R^{\mu v \rho \tau}-R_{\mu v} R^{\mu v}\right) \\
& -\frac{1}{6}\left(\frac{1}{5} R-X\right)_{; \mu}{ }^{\mu}+\frac{1}{2}\left(\frac{1}{6} R-X\right)^{2} .
\end{aligned}
$$

This finite-temperature expansion is precisely the same as the zero-temperature one $[12,13,27]$ times the function $S_{0}^{F}\left(\beta^{2} / t\right)$, which carries the entire temperature dependence. Note that the zero-temperature limit can be reached by taking the limit $\beta \rightarrow \infty$; hence,

$$
\lim _{\beta \rightarrow \infty} H_{\beta}^{(N)}(x, x ; t)=H^{(N)}(x, x ; t) .
$$

Writing the expansion (2.20) as

$$
H_{\beta}^{(N)}(x, x ; t)=\frac{e}{(4 \pi t)^{N / 2}} \sum_{m} a_{m}\left(x ; \beta^{2} / t\right) t^{m}
$$

we can now relate the generalized Seeley coefficients $a_{m}\left(x ; \beta^{2} / t\right)$ to the usual zero-temperature ones by

$$
a_{m}\left(x ; \beta^{2} / t\right)=a_{m}(x) S_{0}^{F}\left(\beta^{2} / t\right),
$$

in complete analogy with the flat-spacetime results [4]. In the above calculation we have found an explicitly covariant result, although the method used was not, since we have treated time and space coordinates on different footing. Nevertheless, at the end of the calculation the spacetime symmetry is completely restored.

\section{CONFORMAL ANOMALY IN $\lambda \phi^{4}$ THEORY}

In this section we discuss a self-interacting scalar field theory in four-dimensional space-time, namely, the $\lambda \phi^{4}$ one, at the one-hoop level in a curved background, at finite temperature. As this is a scalar theory, we need to extend the above discussion on finite-temperature heat kernels to bosonic fields. In order to find the heat-kernel expansion in terms of the Seeley coefficients for these fields we first modify the $\delta$ function

$$
\delta\left[k_{0}-\frac{2 \pi}{\beta}\left(n+\frac{1}{2}\right)\right] \rightarrow \delta\left[k_{0}-\frac{2 \pi n}{\beta}\right]
$$

in Eq. (2.8) as we are now imposing periodic conditions on the scalar fields $\phi\left(x, x_{0}\right)$, when subjected to the translations $x_{0} \rightarrow x_{0}+$ in $\beta$, in Euclidean spacetime,

$$
\phi\left(\mathbf{x}, x_{0}+i n \beta\right)=\phi\left(\mathbf{x}, x_{0}\right) \quad(n=0, \pm 1, \pm 2, \ldots),
$$

following the usual prescriptions of the imaginary-time formalism [11]. The geometrical quantities $V\left(x, x^{\prime}\right)$ and $I\left(x, x^{\prime}\right)$ again are not modified by temperature effects, although here $I\left(x, x^{\prime}\right)$ is regarded as a biscalar. This change in the $\delta$ function implies a modification in the generalized $\Theta$-function transformation (2.11). In fact, this equation for the bosonic case only differs from the spinorial case by the change $n+\frac{1}{2} \rightarrow n$ on the right-hand side (RHS) and by the absence of the factor $(-1)^{n}$ on its LHS. So it is easy to rewrite Eqs. (2.13) and (2.14) for the bosonic case. For example,

$$
S_{0}^{B}(1 / \gamma)=1+2 \sum_{n=1}^{+\infty} \exp \left(-n^{2} / 4 \gamma\right) \text {. }
$$

So the heat-kernel expansion (2.20) is still valid in the bosonic case if we make the substitution $S_{0}^{F}(1 / \gamma) \rightarrow S_{0}^{B}(1 / \gamma)$

The $\lambda \phi^{4}$ theory at the one-loop level is equivalent to its semiclassical approximation in which case we can state [16-18] that

$$
\frac{\lambda}{4 !} \phi^{4} \approx \frac{\lambda}{4} \phi_{c}^{2} \phi^{2}
$$

where we have kept only the quadratic terms in $\phi$, so that the $\phi$ field interacts with its classical counterpart $\phi_{c}$, which behaves like a scalar background field. Including also a gravitational background we write the action as

$S=\frac{1}{2} \int d^{4} x \sqrt{-g}\left(\partial_{\mu} \phi \partial^{\mu} \phi-\frac{1}{6} R \phi^{2}-\frac{\lambda}{2} \phi_{c}^{2} \phi^{2}\right]$,

which is invariant under the Weyl (conformal) transformations

$$
g_{\mu \nu}(x) \rightarrow \exp \{2 \alpha(x)\} g_{\mu \nu}(x)
$$

and

$$
\phi(x) \rightarrow \exp \{-\alpha(x)\} \phi(x) .
$$

To quantize this theory we introduce the generating functional

$$
Z=\int[d \phi] \exp \{-i S\}
$$

which is suitably continued to a curved spacetime with Euclidean signature $(----)$ giving rise to the partition function

$$
Z_{\beta}=\int[d \phi] \exp \left\{-i \int_{0}^{-i \beta} d x_{0} \int d^{3} \mathbf{x} \mathcal{L}_{\text {eff }}\right\},
$$

where the bosonic fields $\phi$ satisfy the periodic condition (3.1). Now, using the operator [18]

$$
D^{2}=\square+\frac{1}{6} R+\frac{\lambda}{2} \phi_{c}^{2}
$$

with a complete set of eigenfunctions $\varphi_{n}$, one can implement Fujikawa's technique [22,23] and show that the anomalous trace of the energy-momentum stress tensor 
$T_{\mu \nu}$, at finite temperature $\beta^{-1}$, is given by

$$
\begin{aligned}
\left\langle T_{\mu}{ }^{\mu}\right\rangle_{\beta} & =\left.\frac{-i}{\sqrt{-g}} \frac{\delta Z_{\beta}}{\delta \alpha(x)}\right|_{\alpha=0} \\
& =-i \sum_{n}\left[\varphi_{n}(x) \varphi_{n}(x)\right]_{\beta} .
\end{aligned}
$$

This divergent quantity must be regularized to yield a finite result. For example, if we use the heat-kernel regularization [30] we obtain

$$
\begin{aligned}
\left\langle T_{\mu}{ }^{\mu}\right\rangle_{\beta}= & \lim _{t \rightarrow 0}\left(\frac{1}{4 \pi}\right)^{2}\left[t^{-2}+t^{-1} a_{1}(x)+a_{2}(x)\right] \\
& \times S_{0}^{B}\left(\beta^{2} / t\right) \\
= & \lim _{t \rightarrow 0}\left(\frac{1}{4 \pi}\right)^{2}\left[t^{-2}+t^{-1} a_{1}(x)+a_{2}(x)\right],
\end{aligned}
$$

which clearly shows the vanishing of the temperature contribution to the anomaly, since

$$
\lim _{t \rightarrow 0} S_{0}^{B, F}\left(\beta^{2} / t\right)=1 \text {. }
$$

This regularization scheme gives a transparent way to visualize the cancellation of the temperature dependence of the anomaly; however, this scheme it is not well suited for the regularization of the anomalous trace, (3.9), since it retains some divergent terms corresponding to $a_{0}(x)$ and $a_{1}(x)$, as can be seen in Eq. (3.10). So if we use this regularization procedure we will also need a renormalization program for physical quantities. To avoid this difficulty we shall use the $\zeta$-function regularization scheme, which gives a finite result for the anomalous trace, without needing an extra renormalization [15].

To this end we define the power kernel $[15,30]$

$K_{\beta}^{(N)}(x, x ; s)=\frac{1}{\Gamma(s)} \int_{0}^{\infty} d t t^{s-1} H_{\beta}^{(N)}(x, x ; t)$,

which is often called a generalized $\zeta$-function, since its trace on $x$ coordinates is equal to the sum over the inverse powers $(-s)$ of the eigenvalues of the operator $D^{2}$. The regularized expression for the anomalous trace (3.9), is then given by

$$
\begin{aligned}
\left.\left\langle T_{\mu}{ }^{\mu}\right\rangle_{\beta}\right|_{\mathrm{reg}} & =-\left.i \sum_{n}\left[\varphi_{n}(x) \varphi_{n}(x)\right]_{\beta}\right|_{\mathrm{reg}} \\
& =\lim _{s \rightarrow 0} \operatorname{tr} K_{\beta}^{(N)}(x, x ; s) .
\end{aligned}
$$

To evaluate this kernel we use the asymptotic expansion of the diagonal part of the heat kernel (2.23) and split the integral appearing in Eq. (3.12) in two parts:

$$
K_{\beta}^{(N)}(x, x ; s)=\frac{e}{(4 \pi)^{N / 2} \Gamma(s)} \int_{0}^{\epsilon} d t t^{s-1-N / 2} \sum_{m} a_{m}\left(x ; \beta^{2} / t\right) t^{m}+\frac{e}{\Gamma(s)} \int_{\epsilon}^{\infty} d t t^{s-1} H_{\beta}^{(N)}(x, x ; t) .
$$

The first integral can also be separated in two parts, using the bosonic version of Eq. (2.24) and Eq. (3.2). The second integral is an analytical function in the entire complex $t$ plane, say, $F_{\beta}(s)$, so

$$
\begin{aligned}
K_{\beta}^{(N)}(x, x ; s)=\frac{e}{(4 \pi)^{N / 2} \Gamma(s)}[ & \int_{0}^{\epsilon} d t t^{s-1-N / 2} \sum_{m} a_{m}(x) t^{m}+(4 \pi)^{-N / 2} F_{\beta}(s) \\
& \left.+2 \int_{\epsilon}^{\infty} d t t^{s-1-N / 2} \sum_{m} a_{m}(x) t^{m} \sum_{n} \exp \left[\frac{-n^{2} \beta^{2}}{4 t}\right]\right] .
\end{aligned}
$$

The second integral in (3.15) vanishes when we take the limit $\epsilon \rightarrow 0$, so we find

$$
K_{\beta}^{(N)}(x, x ; s)=\frac{e s}{(4 \pi)^{N / 2} \Gamma(s+1)}\left[\sum_{m} a_{m}(x) \frac{\epsilon^{m+s-N / 2}}{m+s-N / 2}+(4 \pi)^{-N / 2} F_{\beta}(s)\right] .
$$

Substituting this result in Eq. (3.13) and taking the limit $s \rightarrow 0$, we see that only the term $m=N / 2$ in the sum contributes to the anomaly and that $s F_{\beta}(s)$ also vanishes keeping the anomaly temperature independent:

$$
\left.\left\langle T_{\mu}{ }^{\mu}\right\rangle_{\beta}\right|_{\text {reg }}=\frac{1}{(4 \pi)^{N / 2}} a_{N / 2}(x) \text {. }
$$

Now, using the fact that $\Lambda_{\mu \nu}=0$, since the $\phi$ field is scalar and there is no vector field present, we see that the Seeley coefficient for $N=4$, Eq. (2.21c), reduces to

$$
\begin{aligned}
a_{2}(x)= & \frac{1}{180}\left(R_{\mu \nu \rho \tau} R^{\mu \nu \rho \tau}-R_{\mu \nu} R^{\mu v}\right)-\frac{1}{6}\left(\frac{1}{5} R-X\right)_{; \mu}^{\mu} \\
& +\frac{1}{2}\left(\frac{1}{6} R-X\right)^{2}
\end{aligned}
$$

and the function $X$ is fixed by the operator (3.8),

$$
X=\frac{1}{6} R+\frac{\lambda}{2} \phi_{c}^{2},
$$

so we finally find the anomalous trace for the $\lambda \phi^{4}$ theory in a curved background at finite temperature, in fourdimensional spacetime:

$$
\begin{aligned}
\left.\left\langle T_{\mu}{ }^{\mu}\right\rangle_{\beta}\right|_{\mathrm{reg}}=\frac{1}{16 \pi^{2}} & \frac{1}{180}\left(-\square R-R_{\mu \nu} R^{\mu \nu}+R_{\mu \nu \rho \sigma} R^{\mu \nu \rho \sigma}\right) \\
& \left.+\frac{\lambda}{12} \square \phi_{c}^{2}+\frac{\lambda^{2}}{8} \phi_{c}^{4}\right) .
\end{aligned}
$$

This result coincides with the one appearing in the literature, without taking into account finite-temperature effects $[17,18]$. This temperature independence of the 
anomalous trace is physically understood as a high momentum behavior. This was also proved by $\mathrm{Hu}$ and co-workers [11] by showing that the poles of the effective actions at zero and finite temperature do coincide. In addition, this is an example of the predominance of a local behavior (anomaly) over global behavior (temperature).

\section{CHIRAL SCHWINGER MODEL AT FINITE TEMPERATURE}

In previous attempts to calculate the chiral Schwinger model in curved spacetime [21], the conclusions about the anomalous divergence for the axial-vector current and the dynamically generated mass for the gauge field were not in agreement with the well-accepted flat spacetime results [19]. So before we discuss the finitetemperature case we will discuss this model in curved spacetime at zero temperature. Then, we find the "correct" anomalous divergence and the dynamically generated mass in accordance with the flat spacetime results. Finally we introduce finite-temperature corrections to this model in the case of a curved background.

The chiral Schwinger model in curved background, at zero temperature, can be described by the Lagrangian density $[19-21,31]$

$$
\mathcal{L}=-\frac{1}{4} F_{\mu \nu} F^{\mu v}+i \bar{\psi} \gamma^{\mu}\left[\nabla_{\mu}-i g\left(1-\gamma_{5}\right) A_{\mu}\right] \psi,
$$

where $\nabla_{\mu}=\partial_{\mu}+\frac{1}{2} \omega_{\mu}{ }^{a b} \sigma_{a b}$ is the covariant derivative,

$$
\begin{gathered}
\omega_{a b v}=\frac{1}{2}\left[e_{a}{ }^{\mu}\left(\partial_{\mu} e_{v b}-\partial_{v} e_{\mu b}\right)+e_{a}{ }^{\rho} e_{b}{ }^{\sigma}\left(\partial_{\sigma} e_{\rho c}\right) e_{\mu}{ }^{c}\right. \\
\left.-e_{b}{ }^{\rho} e_{a}{ }^{\sigma}\left(\partial_{\sigma} e_{\rho c}\right) e_{\mu}{ }^{c}\right]
\end{gathered}
$$

is the spin connection and $\sigma_{a b}=\frac{1}{4}\left[\gamma_{a}, \gamma_{b}\right]$ are the generators of the Lorentz group.

Our main problem is to study the behavior of the anomalies in curved spacetime of the chiral Schwinger model using Fujikawa's procedure and the heat-kernel machinery. As is well known the origin of the anomalies, in the path integral formalism, is due to the noninvariance of the fermionic measure under the infinitesimal chiral transformations $[22,23]$

$$
\begin{aligned}
& \psi \rightarrow \psi^{\prime}=\left[1+i g\left(1-\gamma_{5}\right) \varepsilon(x)\right] \psi, \\
& \bar{\psi} \rightarrow \bar{\psi}^{\prime}=\bar{\psi}\left[1-i g\left(1+\gamma_{5}\right) \varepsilon(x)\right] .
\end{aligned}
$$

This problem was solved in flat spacetime looking for the question of the regularization scheme [32]. Following this prescription, we use the hermitian operators

$$
D_{+}^{2}=\not D D^{\dagger} ; \quad D_{-}^{2}=D^{\dagger} \not D,
$$

where

$\not D=\gamma^{\mu}\left[\nabla_{\mu}-i a g\left(1-\gamma_{5}\right) A_{\mu}-i g b\left(1+\gamma_{5}\right) A_{\mu}\right]$,

to regularize, respectively, the infinite sums:

$$
\Sigma_{ \pm}=\sum_{n} \Phi_{n}^{ \pm \dagger}\left(1 \pm \gamma_{5}\right) \Phi_{n}^{ \pm}
$$

where $\Phi_{n}^{ \pm}$are the eigenfunctions of the operators $D_{ \pm}^{2}$. These sums contribute to the Jacobian of the fermionic transformations, as usual in Fujikawa's procedure:
$J(\varepsilon(x))=\exp \left[-\frac{i \eta g^{2}}{\pi} \int d^{2} x e \varepsilon(x) \operatorname{tr}\left(\Sigma_{+}-\Sigma_{-}\right)\right]$,

where $\eta=a-b$. Now, following the standard heat-kernel regularization scheme, we find that

$$
\left.\operatorname{tr} \Sigma_{ \pm}\right|_{\text {reg }}=\lim _{t \rightarrow 0} \operatorname{tr}\left(1 \pm \gamma_{5}\right) H\left(x, x, t D_{ \pm}^{2}\right) .
$$

Taking the limit $t \rightarrow 0$, we see that only the term $a_{1}^{( \pm)}$survives, so that

$$
\left.\operatorname{tr} \Sigma_{ \pm}\right|_{\text {reg }}=\operatorname{tr}\left(1 \pm \gamma_{5}\right) \frac{a_{1}^{ \pm}(x)}{4 \pi}
$$

where the Seeley coefficients $a_{1}^{ \pm}(x)$ are obtained from the heat-kernel expansion (2.21b), in the zero-temperature limit

$$
a_{1}^{ \pm}(x)=\frac{1}{6} R-X^{ \pm} .
$$

Since the operators $D_{ \pm}^{2}$ satisfy the identities

$$
D_{ \pm}^{2}=D_{\mu} D^{\mu}-\frac{1}{4} R+\frac{g}{2 e} \gamma_{5} \epsilon_{\mu \nu} F_{ \pm}^{\mu v},
$$

where $F_{ \pm}^{\mu v}=\partial^{\mu} A_{ \pm}^{v}-\partial^{v} A_{ \pm}^{\mu}$ and $A_{\mu}^{ \pm}=\left(\eta_{\mu v} \pm \epsilon_{\mu v}\right) A^{v}$, we identify $X^{ \pm}=-\frac{1}{4} R+(g / 2 e) \gamma_{5} \epsilon_{\mu \nu} F_{ \pm}^{\mu \nu}$, which leads to

$$
a_{1}^{ \pm}(x)=-\frac{1}{12} R+\frac{g}{2 e} \gamma_{5} \epsilon_{\mu v} F_{ \pm}^{\mu v}
$$

Therefore we have that the difference of the sums appearing in the Jacobian (4.6) reads

$$
\begin{aligned}
\operatorname{tr}\left(\left.\Sigma_{+}\right|_{\text {reg }}-\left.\Sigma_{-}\right|_{\text {reg }}\right) & =-\frac{g \eta}{8 e \pi}\left(\epsilon_{\mu \nu} F_{+}^{\mu v}-\epsilon_{\mu \nu} F_{-}^{\mu v}\right) \\
& =-\frac{g \eta}{\pi} \nabla_{\mu} A^{\mu} .
\end{aligned}
$$

Imposing that the generating functional for the Lagrangian density (4.1), rewritten in terms of the fields $\psi^{\prime}$ and $\bar{\psi}^{\prime}$ defined by the chiral transformation (4.2), does not depend on the parameter $\varepsilon(x)$, we get the anomalous divergence

$$
\nabla_{\mu} J_{5}^{\mu}(x)=-\frac{g \eta}{\pi} \nabla_{\mu} A^{\mu}
$$

In order to obtain the dynamically generated mass for the gauge field, we must consider the finite counterparts of the chiral transformations Eq. (4.2) of the fermionic fields $\psi$ and $\bar{\psi}$. The result of applying these transformations is that the gauge field and the fermions stay decoupled. So iterating the infinitesimal Jacobian (4.6) $N$ times, with $N \rightarrow \infty$, so that $N \varepsilon(x)$ remains finite, we find that the gauge field acquires a mass

$$
m^{2}=\frac{g^{2} \eta}{4 \pi} .
$$

This result is equivalent to the flat spacetime one of Jackiw and Rajaraman [19] when we identify $\eta=2 a^{2} /(a-1)$. The singular cases $\eta=0$ and $\eta \rightarrow \infty$ (or equivalently $a=0$ and $a=1$ ) cannot be obtained as limiting cases of results (4.9) and (4.10) and must be treated separately [20].

Now, we are going to discuss the chiral Schwinger 
model in a curved spacetime at finite temperature. Essentially, we have to compute the sums given by Eq. (4.5), in a thermal average. Following the same steps, as in the case of zero temperature described above, we find

$$
\left.\operatorname{tr}\left(\Sigma_{ \pm}\right)_{\beta}\right|_{\text {reg }}=\lim _{t \rightarrow 0} \operatorname{tr}\left(1 \pm \gamma_{5}\right) H_{\beta}\left(x, x ; t D_{ \pm}^{2}\right) .
$$

Therefore the zero-temperature Jacobian (4.6) can be recalculated here, now taking into account temperature effects. So taking the limit $t \rightarrow 0$ and using Eq. (3.11), as in Sec. III, we find that

$$
\left.\operatorname{tr}\left(\Sigma_{ \pm}\right)_{\beta}\right|_{\mathrm{reg}}=\operatorname{tr}\left(1 \pm \gamma_{5}\right) \frac{a_{1}^{ \pm}(x)}{4 \pi}
$$

where the Seeley coefficients $a_{1}^{ \pm}(x)$ are those given by Eq. (4.11). This result means that the anomalous divergence of the axial-vector current $J_{\mu}^{5}$ for the chiral Schwinger model calculated in a thermal average is the same as the zero-temperature one, Eq. (4.13), as well as the dynamically generated mass for the gauge field, Eq. (4.14).

Naturally, the $\zeta$-function regularization could also have been used here, through the same steps shown in Sec. III, reproducing the above results. As before, the power kernel $K_{\beta}(x, x, \tau)$ is related to the heat kernel $H_{\beta}(x, x ; t)$ by a Mellin transform, Eq. (3.12). Following the steps in Eqs. (3.14) to (3.16), we find that the sums (4.15) reduce again to the zero-temperature ones, Eq. (4.7). In this case, i.e., for the axial anomaly, as is well known, the heat-kernel and $\zeta$-function regularizations lead to the same results, contrary to what happens for the trace anomaly [30].

\section{DISCUSSIONS AND CONCLUSIONS}

In this work we have shown how to relate the generalized Seeley coefficients at temperature $\beta^{-1}$ with the ordinary zero-temperature one in curved spacetime. This relation is exactly the same for flat spacetime. This can be understood by the local character of the Seeley coefficients, although curvature terms are present (since the curvature effects are sensible in the neighborhood of any spacetime point) as well as the inverse temperature $\beta$ as a global effect.
We have also shown that the axial and trace anomalies in curved spacetime remain the same for all finite temperatures. It is worth noting that the cutoff limit $t \rightarrow 0$ is equivalent to the zero-temperature one, where we take $\beta \rightarrow \infty$, so we can state (in $N$ dimensions)

$$
\begin{aligned}
\lim _{\substack{t \rightarrow 0 \\
\beta \rightarrow \infty}} a_{N / 2}\left(x ; \beta^{2} / t\right) & =\lim _{\substack{t \rightarrow 0 \\
\beta \rightarrow \infty}} a_{N / 2}(x) S_{0}^{F}\left(\beta^{2} / t\right) \\
& =a_{N / 2}(x) .
\end{aligned}
$$

Essentially, this mechanism keeps anomalies, in general, free from temperature effects. This can be explained by the high momenta and short wavelengths involved which predominate over global aspects as the temperature. This common feature shared by these anomalies suggests that there is a parallelism between them.

However, the axial anomaly can be determined through the Atiyah-Singer index theorem [33], which states that [22] (for simplicity here, in four-dimensional flat spacetime)

$$
n_{+}-n_{-}=-\frac{1}{16 \pi^{2}} \operatorname{tr} \int d^{4} x^{*} F_{\mu \nu} F^{\mu \nu}
$$

where $n_{ \pm}$are the number of zero modes with chiralities $( \pm)$. So this topological theorem anticipates the temperature independence of the axial anomaly, since finitetemperature effects does not change the topology of the fields. On the other hand, the trace anomaly does not exhibit a topological origin, so the parallel between these two anomalies fails at this point. Thus, it would be desirable to get a deeper insight into the topological aspects that could be related to the trace anomaly.

Finally, let us mention that the technique discussed in this work could also be applied to other anomalies, which have similar structures to those discussed here, such as the gravitational [34] and supercurrent anomalies [35].

\section{ACKNOWLEDGMENTS}

The authors would like to acknowledge the Conselho Nacional de Desenvolvimento Científico e Tecnológico (Brazilian Agency) for partial financial support.
[1] L. Dolan and R. Jackiw, Phys. Rev. D 9, 3320 (1974).

[2] H. Itoyama and A. H. Mueller, Nucl. Phys. B218, 349 (1983).

[3] M. Reuter and W. Dittrich, Phys. Rev. D 32 , 513 (1985); A. Das and A. Karev, ibid. 36, 623 (1987); X.-G. He and G. C. Joshi, ibid. 41, 3796 (1990); T. F. Treml, Can. J. Phys. 68, 96 (1990).

[4] H. Boschi-Filho, C. P. Natividade, and C. Farina, Phys. Rev. D 45, 586 (1992).

[5] J. S. Dowker and R. Critchley, Phys. Rev. D 15, 1484 (1977).

[6] J. S. Dowker and G. Kennedy, J. Phys. A 11, 895 (1978).

[7] G. Kennedy, R. Critchley, and J. S. Dowker, Ann. Phys. (N.Y.) 125, 346 (1980); G. Kennedy, ibid. 138, 353 (1982); J. S. Dowker, Class. Quantum Grav. 1, 359 (1984); R. Camporesi, Phys. Rep. 196, 1 (1990).
[8] K. Kirsten, Class. Quantum Grav. 7, 2169 (1990).

[9] I. Drummond, Nucl. Phys. B190 [FS3], 93 (1981).

[10] G. Semenoff and D. Weiss, Phys. Rev. D 31, 689 (1985).

[11] B. L. Hu, Phys. Lett. 108B, 19 (1982); B. L. Hu, R. Critchley, and A. Stylianopoulos, Phys. Rev. D 35, 510 (1987).

[12] J. Schwinger, Phys. Rev. 82, 664 (1951); B. S. De Witt, in Relativity, Groups and Topology II, Proceedings of the Les Houches Summer School, Les Houches, France, 1983, edited by B. S. De Witt and R. Stora, Les Houches Summer School Proceedings Vol. 40 (North-Holland, Amsterdam, 1984).

[13] N. D. Birrell and P. C. W. Davies, Quantum Fields in Curved Space (Cambridge University Press, Cambridge, England, 1982).

[14] C. Bernard, Phys. Rev. D 9, 3312 (1974).

[15] S. W. Hawking, Commun. Math. Phys. 55, 133 (1977). 
[16] I. T. Drummond and G. M. Shore, Phys. Rev. D 19, 1134 (1979); J. Guven, Phys. Rev. D 35, 2378 (1987).

[17] M. A. Valle and M. A. Goñi, Phys. Rev. D 36, 615 (1987).

[18] M. S. Alves and J. Barcelos-Neto, Mod. Phys. Lett. A 4, 155 (1989).

[19] R. Jackiw and R. Rajaraman, Phys. Rev. Lett. 54, 1219 (1985).

[20] H. O. Girotti, H. J. Rothe, and K. D. Rothe, Phys. Rev. D 33, 514 (1986).

[21] J. Barcelos-Neto and A. Das, Z. Phys. C 32, 527 (1986).

[22] K. Fujikawa, Phys. Rev. Lett. 42, 1195 (1979); Phys. Rev. D 21, 2848 (1980).

[23] K. Fujikawa, Phys. Rev. Lett. 44, 1733 (1980).

[24] T. S. Bunch and L. Parker, Phys. Rev. D 20, 2499 (1979).

[25] R. T. Seeley, Am. Math. Soc. Proc. Symp. Pure Math. 10, 288 (1967).

[26] In Drummond's paper [9], which also deals with quasiequilibrium conditions, our Eq. (2.7) appears without mentioning the geometrical quantities $V\left(x, x^{\prime}\right)$ and $I\left(x, x^{\prime}\right)$. This is so because he did a scale transformation in the fields which absorbed these factors. Doing the inverse transformation one arrives straightforwardly at our Eq. (2.7).
[27] S. Yajima, Class. Quantum Grav. 5, L207 (1988).

[28] B. Davies, Integral Transforms and Their Applications (Springer-Verlag, New York, 1978); G. G. MacFarlane, Philos. Mag. 40, 188 (1949).

[29] S. M. Christensen, Phys. Rev. D 14, 2490 (1976).

[30] M. S. Alves, C. Farina, and C. Wotzasek, Phys. Rev. D 43, 4145 (1991).

[31] Our conventions are $\eta_{\mu \nu}=\operatorname{diag}(1,-1), \quad \epsilon^{01}=1$, $\left\{\gamma^{\mu}, \gamma^{v}\right\}=2 \eta^{\mu v}, \quad \gamma^{\mu}=\gamma^{0} \gamma^{\mu^{\dagger}} \gamma^{0}, \quad \gamma^{\mu} \gamma^{v}=\eta^{\mu v}+\epsilon^{\mu v} \gamma_{5}$, $\gamma_{5}=\gamma^{0} \gamma^{1}, \sigma_{\mu \nu}=1 / 4\left[\gamma_{\mu}, \gamma_{\nu}\right], \gamma_{5} \gamma_{\mu}=\epsilon_{\mu \nu} \gamma^{\nu}$.

[32] J. Barcelos-Neto and C. Farina de Souza, Phys. Rev. D 38, 613 (1988)

[33] M. Atiyah and I. Singer, Ann. Math. 87, 484 (1968); M. Atiyah, R. Bott, and V. Patodi, Invent. Math. 19, 279 (1973).

[34] L. Alvarez-Gaumé and E. Witten, Nucl. Phys. B234, 269 (1983).

[35] M. T. Grisaru, in Recent Developments in Gravitation, Proceedings of the NATO Advanced Study Institute, Cargese, France, 1978, edited by S. Deser and M. Levy, NATO, ASI Series B: Physics Vol. 44 (Plenum, New York and London, 1978); H. Suzuki, Phys. Rev. D 33, 2948 (1986). 